\title{
Imaging of Single Vacancies and Dopant Complexes in Aluminum Nitride
}

\author{
Tim B. Eldred ${ }^{1}$, Joshua S. Harris ${ }^{1}$, Jonathon N. Baker ${ }^{1}$, Douglas L. Irving ${ }^{1}$ and James M. LeBeau ${ }^{1}$ \\ ${ }^{1}$ North Carolina State University, Materials Science and Engineering, Raleigh, USA
}

To enable efficient aluminum nitride UV LEDs and power electronics, charge carrier concentration must be carefully engineered via dopant control. Silicon is the most studied and promising dopant in AlGaN systems, however it has been demonstrated that charge carrier concentration drops as Al content increases [1,2]. The prevailing explanation for the decrease in charge carrier concentration is that other defects compensate Si donors at high Si concentrations. The exact distribution and nature of the defects responsible have not been identified, however it is theorized that a complex with cation vacancies contribute to the effect [3]. The purpose of this study is to determine a method of identifying and mapping the vacancies and other defects in the material for the purpose of studying defect formation and confirming the statistical models used to guide growth techniques.

In this presentation, we will discuss how high precision and accuracy scanning transmission electron microscopy (STEM) can be used to detect and map dopant complexes formed during metal-organic chemical vapor deposition crystal growth, specifically cation vacancies complexed with cation substitution dopants. We will present the approach to model the structure using DFT calculations in the Vienna ab initio Simulation Package (VASP 5.3.4). Specifically, formation energies determined using the grand canonical formalism are used to determine the relative concentrations of the different potential configurations of the $\mathrm{Si}_{\mathrm{Al}}-\mathrm{V}_{\mathrm{Al}}$ complexes. Multislice simulations are then performed using the various calculated defect coordinates as a function of sample thicknesses and operating condition.

To observe the effect of the defect, intensities and positions are then extracted from ADF STEM images as shown in Figure 1. ADF STEM with a detector inner semiangle of $28 \mathrm{mrad}$ was used to maximize the signal from the weakly scattering aluminum and nitrogen. For a Si-vacancy complex, the relative intensity of the column decreases by an amount relative to the thickness of the sample as well as the scattering factor of the atoms in the column. Because the intensity change is $>4 \%$ due to the vacancy, we will discuss how these results, well within the $\sim 1 \%$ minimum detection threshold [4], can be combined with displacements to unambiguously identify these defect complexes. We will show that the apparent atom column positions near the vacancy, as in Figure 1(right), also contract around the Al vacancy. We will further discuss how a single vacancy can be discriminated from the defect complex by the magnitude of the displacements. For example, as shown in Figure 2, the displacement of atom columns compared to the 'bulk' regions is higher between nitrogen atom columns in comparison to aluminum. Finally, we will highlight how local distortion and intensity measurements provide the framework for detection and classification of single atom defect and vacancy complexes in nitride materials [5].

References:

[1] S.F. Chichibu, H. Miyake, Y. Ishikawa, J. Appl. Phys. 113 (2013)

[2] Y. Taniyasu, M. Kasu, N. Kobayashi, Appl. Phys. Lett. 81 (2002) 1255-1257.

[3] A. Uedono, K. Tenjinbayashi, T. Tsutsui, J. Appl. Phys. 111 (2012)

[4] J. Feng, A.V. Kvit, C. Zhang, Microsc. Microanal. 22 (2016) 902-903.

[5] The authors acknowledge funding from AFOSR FA9550-17-1-0225 

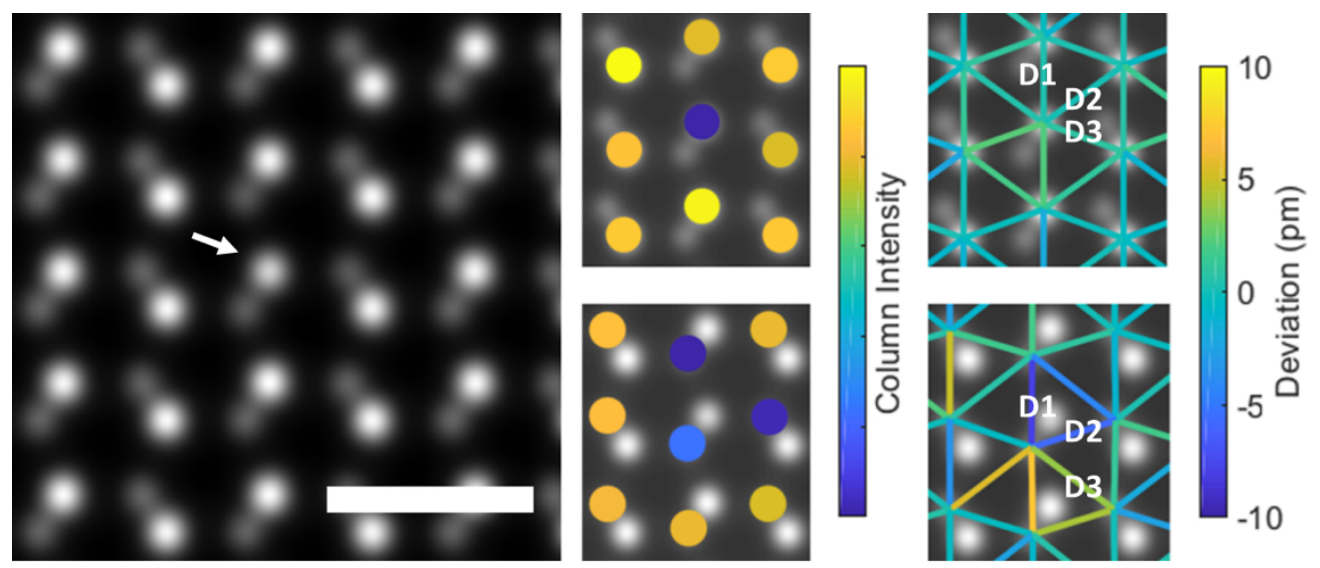

Figure 1. Simulated vacancy complex in a $3 \mathrm{~nm}$ sample (left, vacancy indicated by arrow) (scale bar - 5 $\AA$ ). Aluminum and nitrogen column intensities (center), showing the intensity decrease at and around the vacancy. Deviations in column-column distance (right) between aluminum and nitrogen columns around the vacancy.
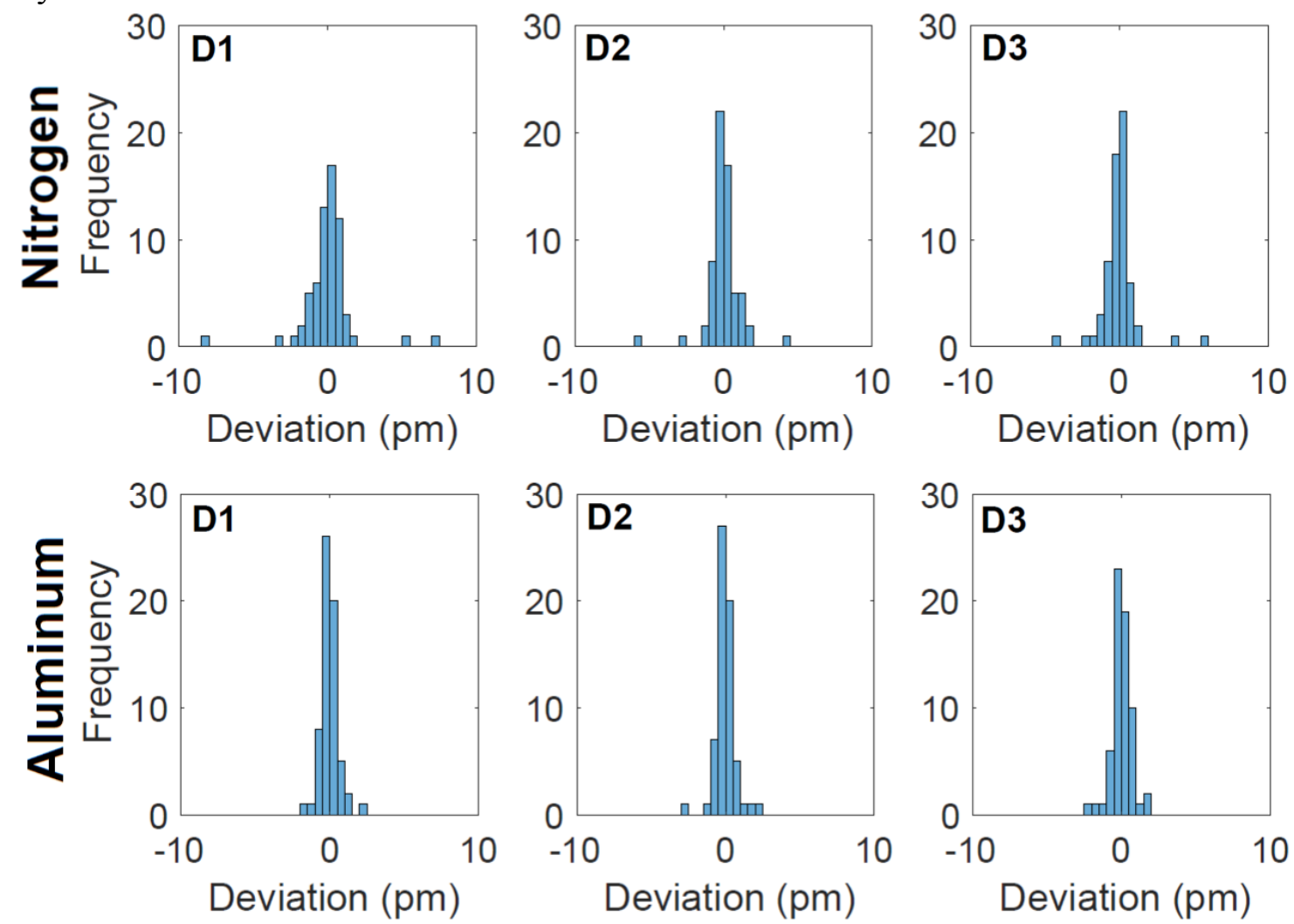

Figure 2. Distribution of displacements from the average atom column to atom column separation. D1, D2, D3 distances are highlighted in Figure 1 (right). Outliers in the nitrogen atom column distributions should be readily observed in experiment. 